\title{
Global distribution pattern of anthropogenic nitrogen oxide emissions: Correlation analysis of satellite measurements and model calculations
}

\author{
N. Toenges-Schuller, ${ }^{1}$ O. Stein, ${ }^{1}$ F. Rohrer, ${ }^{1}$ A. Wahner, ${ }^{1}$ A. Richter, ${ }^{2}$ J. P. Burrows, ${ }^{2}$ \\ S. Beirle, ${ }^{3}$ T. Wagner, ${ }^{3}$ U. Platt, ${ }^{3}$ and C. D. Elvidge ${ }^{4}$ \\ Received 12 April 2005; revised 16 November 2005; accepted 13 December 2005; published 11 March 2006.
}

[1] Nitrogen oxides play a key role in tropospheric chemistry; to study the distribution patterns of the corresponding anthropogenic emissions (fossil, industrial, waste), we use three independent data sources: GOME measurements of the tropospheric $\mathrm{NO}_{2}$ column density fields, the EDGAR 3 emission inventory as an estimation of the anthropogenic $\mathrm{NO}_{\mathrm{x}}$ emissions and nighttime images of worldwide human settlements seen by the DMSP OLS satellite instrument as a proxy for these emission patterns. The uncertainties are not known precisely for any of the fields. Using the MOZART-2 CTM, tropospheric column density fields are calculated from the emission estimates, and transformations are developed to turn the GOME columns into anthropogenic emission fields. Assuming the errors of the three data sources (GOME, EDGAR, lights) to be independent, we are able to determine ranges for the pattern errors of the column density fields and values for the pattern errors of the source fields by a correlation analysis that connects relative error (co)variances and correlation coefficients. That method was developed for this investigation but can generally be used to calculate relative error variances of data sets, if the errors of at least three of them can be assumed to be independent. We estimate the pattern error of the EDGAR 3 anthropogenic $\mathrm{NO}_{x}$ emission field as $(27 \pm 5) \%$, which can be reduced by combining all fields to $(15 \pm 3) \%$. By determining outliers, we identify locations with high uncertainty that need further examination.

Citation: Toenges-Schuller, N., O. Stein, F. Rohrer, A. Wahner, A. Richter, J. P. Burrows, S. Beirle, T. Wagner, U. Platt, and C. D. Elvidge (2006), Global distribution pattern of anthropogenic nitrogen oxide emissions: Correlation analysis of satellite measurements and model calculations, J. Geophys. Res., 111, D05312, doi:10.1029/2005JD006068.

\section{Introduction}

[2] For a long time, the global distribution of nitrogen oxides $\left(\mathrm{NO}_{\mathrm{x}}=\mathrm{NO}+\mathrm{NO}_{2}\right)$ could only be analyzed by model calculations, using global chemistry transport models (CTMs), because ground-based or air-borne measurement campaigns were necessarily temporally and spatially limited. As another approach, now satellite instruments can measure atmospheric trace gas concentrations with global coverage and fixed spatial and temporal resolution over a long period of time. Since its launch in April 1995, the satellite instrument GOME has been measuring spectra in the UV and visible range that allow the retrieval of a range of trace gases including $\mathrm{NO}_{2}$. Satellite measurements and model calculations can easily be compared, as in both cases mean values over a certain area are determined: The satellite instrument measures the absorption along the complete light path and model outputs are mean values of the grid boxes given by the resolution of the model, whereas

\footnotetext{
${ }^{1}$ ICG-II, Forschungszentrum Jülich, Jülich, Germany

${ }^{2}$ IUP, University of Bremen, Bremen, Germany.

${ }^{3}$ IUP, University of Heidelberg, Heidelberg, Germany.

${ }^{4}$ National Geophysical Data Center, NOAA, Boulder, Colorado, USA.
}

Copyright 2006 by the American Geophysical Union. 0148-0227/06/2005JD006068\$09.00 for in situ measurements it is difficult to decide whether point measurements are representative for the corresponding grid box of a global model. Unfortunately, both, model calculations and satellite measurements may contain large uncertainties which are difficult to estimate. Model errors include emissions, transport and chemistry, and models are not evaluated globally but only by comparison with measurement campaigns or single stations. For the retrieval of tropospheric trace gas concentrations from satellite spectra, assumptions of local parameters such as cloud coverage, surface albedo, and vertical profile of the retrieved trace gas are necessary, which are often gained by modeling.

[3] In this analysis, we use GOME retrievals independent of model calculations, the EDGAR 3 database that estimates anthropogenic emissions based on economic and population density data of the different countries, and the MOZART-2 global CTM. As an independent data source, we use nighttime images of human settlements in the visible range of the satellite instrument DMSP-OLS (defense meteorological satellite program, optical linescan system) as a proxy for the pattern of the anthropogenic emissions. The aims of this analysis are the quantification of pattern errors of the EDGAR 3 anthropogenic $\mathrm{NO}_{\mathrm{x}}$ emissions (excluding biomass burning and agriculture) and the GOME measurements in the area dominated by anthropogenic emissions, the construction of an optimized emission field, and the 
identification of outliers in the data sets to indicate areas where the disagreements between the fields and therefore the uncertainties are highest. The assumptions made in this paper to achieve these aims are purely statistical and different from the assumptions made in the GOME retrievals and models.

\section{2. $\mathrm{NO}_{2}$ Columns}

[4] In this section, $\mathrm{NO}_{2}$ column density fields are described and a correlation analysis connecting spatial correlation coefficients and relative error variances is carried out.

\subsection{GOME Measurements}

[5] The GOME (Global Ozone Monitoring Experiment) instrument [Burrows et al., 1995] is situated onboard the second European remote sensing satellite ERS-2, which was launched April, 21st 1995. The satellite passes the equator in a sun-synchronous polar orbit at 10:30 local time and reaches global coverage at the equator after three days. The instrument itself consists of four spectrometers with 1024 channels each in the UV and visible range. It operates in nadir geometry, which means it is looking straight down to the earth measuring the sunlight scattered back from the earth and from the air column above.

\subsubsection{Retrieval}

[6] One of the data products retrieved from the GOME measurements are tropospheric $\mathrm{NO}_{2}$ column densities. Retrievals have been carried out by Wenig [2001], Leue et al. [2001], Richter and Burrows [2002], Martin et al. [2002, 2003], Nüß [2005], Boersma et al. [2004], and Beirle et al. [2004]. The main steps from absorption spectra to tropospheric column densities are: Calculation of the so called slant column densities, separation of the stratospheric and the tropospheric part of the columns, and conversion of the slant columns into vertical columns. They are described in detail in the publications cited above. The slant column densities are calculated using the DOAS method [Platt et al., 1979] using Lambert-Beer's law, and give the integral of the $\mathrm{NO}_{2}$ along the lightpath from the sun through the atmosphere of the earth scattered back to GOME. In particular in the presence of clouds or aerosols, multiple scattering also plays a role. The stratospheric part of the $\mathrm{NO}_{2}$ can be estimated using the reference sector method (RSM), an image processing algorithm (IPA) or calculated by a model of the stratosphere. For the RSM, the stratospheric $\mathrm{NO}_{2}$ is assumed to have only a weak longitudinal dependency. At each latitude, the columns in an area remote of the known sources are assumed to be of stratospheric origin and subtracted from the total columns. In the IPA it is assumed that the columns over the oceans over cloudy pixels are of stratospheric origin. The pattern of these pixels is extrapolated over the continents. To convert slant column densities into vertical column densities the so called air mass factor (AMF), the ratio of the two quantities, is applied. The AMF depends on the observing geometry (scanning angle and solar zenith angle), but also on the vertical profile of the absorbing trace gas, surface albedo, cloud coverage, cloud height, and aerosol loading. It is usually calculated by a radiative transfer model. Parameters as vertical profile and aerosols are often calculated by a chemical transport model (CTM) of the troposphere.

\subsubsection{GOME Leue and GOME Richter}

[7] For the analysis carried out in this paper it is essential that the errors of the fields examined can be assumed to be independent. Since we will also examine model calculations from the global CTM MOZART-2 (see below), we used only GOME retrievals that were done without use of a CTM to calculate the vertical profile of $\mathrm{NO}_{2}$ for the AMF. This leaves three GOME retrievals: Leue et al. [2001], Richter and Burrows [2002], and Beirle et al. [2004]. The main advantage in the latter retrieval is the high resolution of $80 \mathrm{~km} \times 40 \mathrm{~km}$ (a special narrow observing mode of GOME was used), which is paid for by a longer time to reach global coverage and less data for calculating mean values. As the resolution of this analysis is limited by the model resolution $\mathrm{T} 63\left(\approx 1.87^{\circ}\right.$ lon $\times$ $1.89^{\circ}$ lat), this advantage is not relevant here. So in this paper, we will use the two earliest GOME retrievals: by Leue et al. [2001], henceforth called GOME Leue, and by Richter and Burrows [2002], henceforth called GOME Richter. Both data sets consist of monthly mean values for the period from 1996 to 2001 with a spatial resolution of $0.5^{\circ}$ lon $\times 0.5^{\circ}$ lat. Both groups used the DOAS technique [Platt et al., 1979] to retrieve the slant column densities and to avoid a CTM, and both groups assumed the tropospheric vertical profile of $\mathrm{NO}_{2}$ to be independent of season and location. Leue et al. [2001] applied an IPA to separate tropospheric and stratospheric $\mathrm{NO}_{2}$. They used all pixels regardless of cloud coverage but applied a global factor of four to correct for the influence of the clouds. Richter and Burrows [2002] used the RSM for the separation of the stratospheric $\mathrm{NO}_{2}$; their reference sector is placed over the Pacific Ocean between $170^{\circ} \mathrm{W}$ and $180^{\circ} \mathrm{W}$ longitude. They used only pixels with a cloud coverage of less then $10 \%$, so the influence of clouds on radiative transfer is small. Therefore, their data set contains undefined values where the cloud coverage was too high on too many days in a given month.

\subsubsection{Annual Mean Values}

[8] In the following, correlations between annual mean fields of the various data sets are determined at the resolution of the MOZART-2 model (T63, see below). Therefore, the monthly values were averaged and the resolution was changed. To deal with the gaps at undefined values in the GOME Richter data set, at first a mean field for each of the seven years from 1996 to 2001 was calculated. To avoid a bias due to the annual variability, a pixel was assigned a defined value only if the pixel was defined in all months of that year. Then we averaged over the seven annual mean fields. By this, the gaps could partly be filled if they were defined in the mean of at least one of the years. After that the resulting field was interpolated to the resolution T63. In this step, a pixel was assigned a defined value only if at least $50 \%$ of the area of the pixel was covered with defined values in the original resolution.

\subsection{MOZART-2 Model Calculations}

[9] MOZART (Model for OZone And Related chemical Tracers) is a global 3D CTM developed at NCAR (National Center of Atmospheric Research) in Boulder, Colorado, NOAA/GFDL (General Fluid Dynamics Laboratory) in Princeton, New Jersey, and at the Max Planck Institute for Meteorology in Hamburg, Germany. The current version 
MOZART-2 is published [Horowitz et al., 2003] and released as a community model.

[10] MOZART-2 simulates the mixing ratios of 63 chemical species from the surface of the earth up to the lower stratosphere. The chemical mechanism contains 167 chemical and photochemical reactions. The calculations for this study were done with a spatial resolution of T63 $\left(\approx 1.87^{\circ}\right.$ lon $\times 1.89^{\circ}$ lat $), 31$ hybrid $\sigma$-levels as vertical coordinate, and a time step of 15 minutes. As meteorological constraint, analysis files from the European Centre for Medium-Range Weather Forecast (ECMWF) of the year 1995 were used.

[11] Especially for short-lived species such as $\mathrm{NO}_{2}$, a crucial part of a CTM is the emission inventory used. MOZART emissions are based on the EDGAR 3 inventory (see section 2.2.1) with additions and modifications as described by Horowitz et al. [2003]. A second model run was done, for which the anthropogenic $\mathrm{NO}_{\mathrm{x}}$ emissions were exchanged by a source based on satellite images of the nighttime lights of the world, see section 2.2.2. In this paper, we use the term anthropogenic as in the input files for the MOZART model: by anthropogenic emissions we mean fossil sources (energy and traffic), industrial processes and waste; due to their different variations in time, anthropogenic biomass burning (often hard to distinguish from wildfires) and agricultural emissions are part of the biogenic (not necessarily natural) emissions.

\subsubsection{Emission Inventory: EDGAR 3}

[12] The current version of the emission inventory EDGAR 3 (Emission Database for Global Atmospheric Research) is an estimate for the year 1995 and was described in detail by Olivier et al. [2001]. The main steps for estimating anthropogenic emissions are the following: Compilation of economic activity data for all countries of the world, transformation of these activity data to emissions by use of national or regional emissions factors for each included trace gas and finally mapping these emissions to a geographical grid of the resolution $1^{\circ} \times 1^{\circ}$ by use of gridded activity data maps. As default population density maps (urban, rural) were used for fuel combustion, except for power plants and industries in Europe and except for steel and cement plants globally, for which point source maps were used. The withincountry distribution of large scale biomass burning emissions (forests and savannahs) were based on a ATSR satellite data for fire counts in 1997. The uncertainty for the estimation of the global source strength of the anthropogenic $\mathrm{NO}_{\mathrm{x}}$ emissions is stated to be about $50 \%$ ( $95 \%$ confidence interval, $25 \%$ at one standard deviation), mainly due to the uncertainty in road transport and large scale biomass burning emissions.

\subsubsection{OLS: Nighttime Lights of the World}

[13] As an independent distribution pattern, satellite images of the Operational Linescan System (OLS, part of the Defense Meteorological Satellite Program of the U.S. Air Force) [Dubach and $N g, 1988$ ] shall be used as a proxy for the anthropogenic $\mathrm{NO}_{\mathrm{x}}$ emissions. Images from October 1994 until March 1995 were evaluated by Elvidge et al. [2001], who constructed global maps with high resolution $(0.5 \mathrm{~km} \times 0.5 \mathrm{~km})$ of human settlements, fires, gas flares and heavily lit fishing boats according to the frequency of occurrence and the geographical location of the detected lights. The detector settings are such that even very weak lights can be detected. The disadvantage of this is that for a medium-sized town the detector is already saturated. So the data product does not contain light intensities but for each pixel the percentage of observations when this pixel was detected as lighted. Most cities are detected at $90 \%-100 \%$ of all observations, but small villages $(\approx 200$ inhabitants) are detected in only $10 \%-20 \%$ of the observations [Elvidge et al., 2001]. Doll et al. [2000] analyzed country-total relationships between the lighted area of the human settlements and socioeconomic parameters such as population density, national gross product, electrical energy consumption and $\mathrm{CO}_{2}$ emissions. For the country-total relationship between lighted area of human settlements and $\mathrm{CO}_{2}$ emissions per country from EDGAR 2 they found a correlation coefficient of $R=0.89$. Significant outliers were countries such as Russia, China or North Korea, which according to the authors is due to less bright street lighting in centrally planned economies.

[14] We will use the lights of the human settlements as a proxy for the pattern of the anthropogenic $\mathrm{NO}_{\mathrm{x}}$ emissions (fossil, industrial and waste, see section 2.2): Much light indicates the existence of many people with a certain industrial infrastructure who are emitting much $\mathrm{NO}_{\mathrm{x}}$. To turn the lights into $\mathrm{NO}_{\mathrm{x}}$ emissions for the model we integrated over all lighted pixels in each T63 box; to get an emission density the result was divided by the total area of the box. Due to the summation over more than 3500 pixels, an originally discrete quantity (percentage of lighted observations, in most cases $100 \%$ ) turns into a quasi continuous quantity, as an emission field should be. We assumed the anthropogenic emissions to have no annual variability, which seems an acceptable approximation since we will only analyze annual mean fields later. The field was scaled with the total source strength of the anthropogenic EDGAR $3 \mathrm{NO}_{\mathrm{x}}$ emissions, so the distribution pattern of the light-based source is independent of EDGAR, but not the global source strength.

[15] At the resolution T63, the correlation coefficient between EDGAR anthropogenic (without biomass burning and agriculture) and the light source is $R=0.79$. Assuming no common errors, $R^{2}=62 \%$ of common variance originate in the true field contained in both. As the correlation of the GOME retrievals to the light field is as good as that to EDGAR (see Table 4), the remaining 38\% variance of the light field cannot entirely be caused by errors. This will be quantified in section 3.2. Thus, at the resolution T63, a combination improving both fields is possible and will be carried out in section 3.3.

\subsubsection{Model Column Densities}

[16] Both with the standard model source files (based on EDGAR 3) and with source files where the anthropogenic $\mathrm{NO}_{\mathrm{x}}$ emissions were replaced by the light-based source, a MOZART-2 model calculation of one and a half years was done, of which only the last year was used to avoid contamination by the spin-up in the model results.

[17] For better comparison with the GOME fields, a model output at 10:30 local time (i.e. the GOME overpass time) was used. Tropospheric column densities were calculated by integrating from the surface to the thermal tropopause $[W M O, 1957]$. We did not use the RSM as in the GOME retrieval by Richter and Burrows to avoid common 
Table 1. Spatial Correlation Coefficients $R_{i j}$ of the Four Column Density Fields $X_{i}{ }^{\text {a }}$

\begin{tabular}{lccccccc}
\hline & \multicolumn{3}{c}{ All Grid Points Defined } & & \multicolumn{3}{c}{ Restricted to Examined Area } \\
\cline { 2 - 4 } Spatial Correlation & MOZART Lights & GOME Richter & GOME Leue & & MOZART Lights & GOME Richter & GOME Leue \\
\hline MOZART EDGAR & $0.91 \pm 0.01$ & $0.72 \pm 0.01$ & $0.63 \pm 0.02$ & & $0.84 \pm 0.02$ & $0.71 \pm 0.03$ & $0.57 \pm 0.03$ \\
MOZART lights & & $0.72 \pm 0.01$ & $0.66 \pm 0.02$ & & & $0.69 \pm 0.03$ & $0.61 \pm 0.03$ \\
GOME Richter & & & $0.85 \pm 0.01$ & & $0.86 \pm 0.01$ \\
\hline
\end{tabular}

aStated errors: one standard deviation, gained by the bootstrap method, left: using all grid points defined in each data set, right: restricted to examined area (see section 2.3.1).

errors between the two data fields. For the global annual mean tropospheric $\mathrm{NO}_{2}$ column density field, the modeled local time $(10: 30)$ values are highly correlated to corresponding columns based on daily mean values $(R=$ 0.99 ), so for correlation analyses, it is not critical whether daily mean values or local time values are used.

[18] In Table 1 (left) the spatial correlation coefficients of the two model calculations with EDGAR and the lights source and the two GOME retrievals at the grid points defined in all sets are listed. The high correlations of the model calculation with the anthropogenic $\mathrm{NO}_{\mathrm{x}}$ source based on the lights with the satellite fields again justify the use of the lights as proxy for anthropogenic emissions.

[19] Savage et al. [2004] correlated annual mean fields of the tropospheric $\mathrm{NO}_{2}$ column densities of the year 1997 of GOME measurements and a model calculation by the global CTM TOMCAT. They found a spatial correlation coefficient of $R=0.79$. The higher correlation can be explained by the GOME retrievals they used: The tropospheric vertical profile of the $\mathrm{NO}_{2}$ used in the retrieval was calculated by the same model TOMCAT the comparison was done with later, and the subtraction of the stratospheric part of the columns was done both for model and satellite by a combination of RSM and stratospheric model. This self consistent comparison allows the examination of errors outside the assumptions made during the retrieval. In this paper, however, we want to follow a different approach: We want to minimize the common errors between the examined fields to be sure that the common variance of two fields is due to common truth rather than to common errors.

[20] Since several direct comparisons between GOME measurements and model calculations are published already [e.g., Velders et al., 2001; Lauer et al., 2002; Savage et al., 2004], in this paper, the fields shall be compared by correlation analysis, with a purely statistical approach to the errors. For a detailed discussion of GOME retrieval errors, see, e.g., Boersma et al. [2004] and for model errors see, e.g., Savage et al. [2004].

\subsection{Correlation Analysis}

[21] The correlation analysis, as described in Appendix A, connects correlation coefficients with relative error variances and covariances. It will be performed in those areas dominated by anthropogenic emissions for the four fields $X_{1}$ : MOZART EDGAR, $X_{2}$ : MOZART lights, $X_{3}$ : GOME Richter, $X_{4}$ : GOME Leue. For these column density fields, error ranges will be given.

\subsubsection{Area of the Analysis}

[22] In the following, the global view is narrowed to those areas dominated by anthropogenic emissions, because here two independent estimations of the source field are available: EDGAR 3 and the lights. A second requirement is that a grid point must be placed over land, since $\mathrm{NO}_{2}$ columns over the oceans will be dominated by transport rather than by emissions. To include various coastal cities, a grid point is taken into account if at least $10 \%$ of its area is land area. Furthermore, all fields must have defined values in the whole area of the analysis. To sum up, the area of the analysis contains all grid points where (1) all four fields have defined values, (2) $\frac{1}{2}$ anthropogenic emissions $($ EDGAR + lights $)>$ other emissions EDGAR, and (3) land fraction of area $>10 \%$. This leaves 1463 out of 18432 grid points. The spatial correlation coefficients in this area are listed in Table 1 (right), the masked fields are shown in Figure 1.

\subsubsection{Pattern Errors: Ranges}

[23] As shown in Appendix A, pattern errors $\left(e_{i i}, e_{i j}\right)$ and correlation coefficients $\left(R_{i j}\right)$ of data sets are connected by equation (A5):

$$
R_{i j}^{2}\left(1-e_{i j}\right)^{2}=\left(1-e_{i i}\right)\left(1-e_{j j}\right)
$$

with $e_{i i}$ and $e_{i j}$ defined as the relative error variances and covariances, respectively. For four fields, this equation represents a system of six equations with ten unknown variables: The pattern errors of the four fields $e_{i i}$ and the six relative error covariances $e_{i j}$ between the fields. There will surely be common errors between the two model calculations because the MOZART errors are common in both fields. Likewise, there will be common errors between the two satellite retrievals because the data come from the same instrument and the evaluation algorithms are not independent. To assume independence of the errors between the model calculations on the one hand and the satellite retrievals on the other hand seems plausible: $e_{13}=$ $e_{14}=e_{23}=e_{24}=0$. However, it is not possible to solve the six equations for the remaining six variables: The determinant of the system becomes zero in that case which means there are either no solutions or an infinite number. Solving the system for the four relative error covariances yields:

$$
\frac{R_{23} R_{14}}{R_{13} R_{24}}=\frac{\left(1-e_{13}\right)\left(1-e_{24}\right)}{\left(1-e_{23}\right)\left(1-e_{14}\right)} .
$$

If all the error covariances are zero, the ratio of the correlation coefficients on the left side of the equation must be one, which means there is one degree of freedom left and leaves an infinite number of solutions. If the ratio 

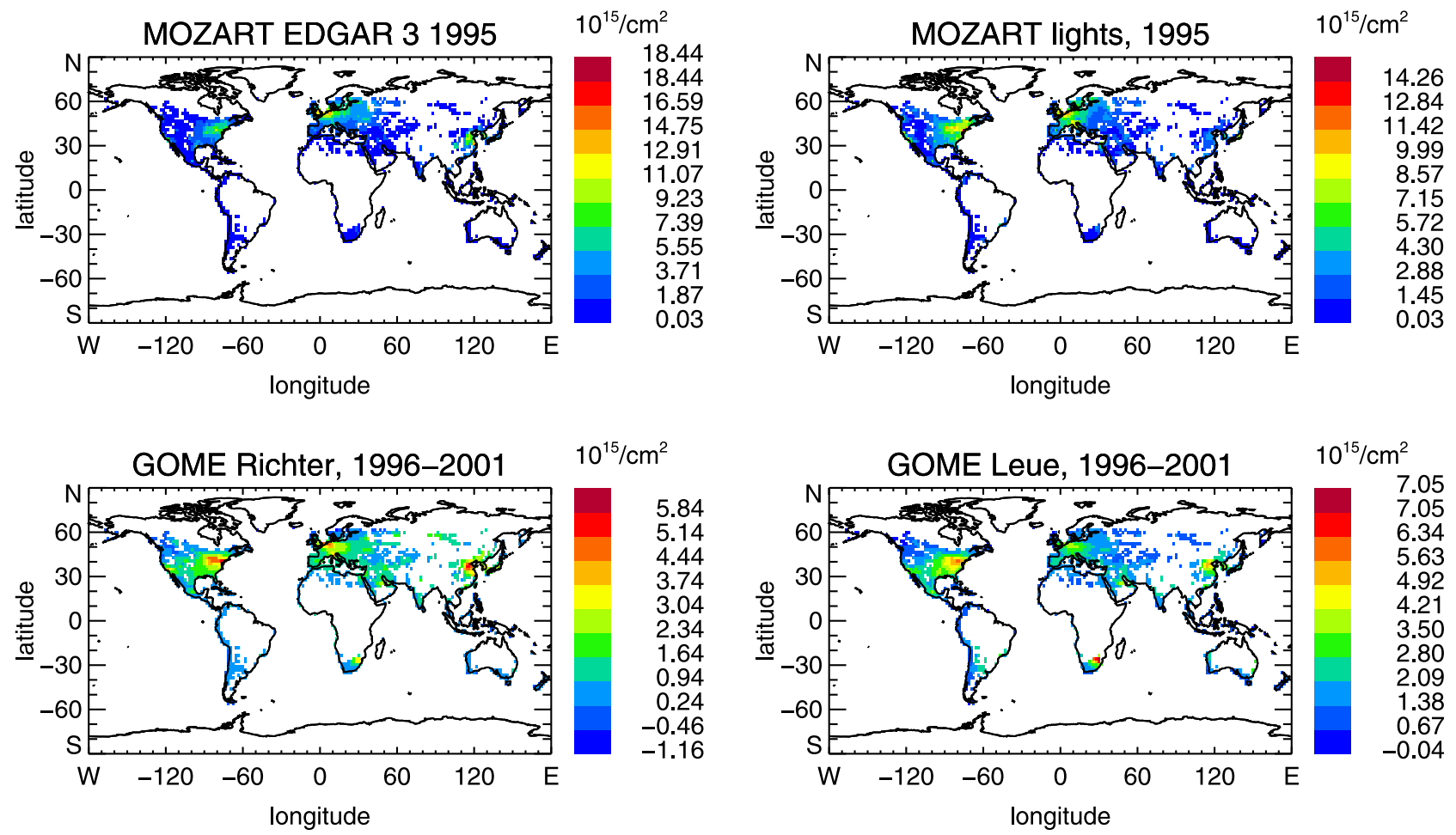

Figure 1. Annual mean fields of tropospheric $\mathrm{NO}_{2}$ column densities, MOZART model calculations, and GOME measurements; color scale linear from minimum to maximum for each field to compare patterns rather than absolute values; the area not analyzed is masked white.

significantly differs from one, the system is inconsistent with the assumptions and there are no solutions. Inserting the correlation coefficients from Table 1 (right), equation (1) yields:

$$
\frac{R_{23} R_{14}}{R_{13} R_{24}}=0.92 \pm 0.02
$$

the error range was determined by the bootstrap method [Efron, 1982], and corresponds to one standard deviation. Thus, the assumption $e_{13}=e_{14}=e_{23}=e_{24}=0$ is not consistent with equation (A5) and the correlation coefficients in Table 1 (right). So we changed the assumption: We suppose the errors between the model calculations on the one hand and the satellite retrievals on the other hand to be as independent as possible. Therefore, the relative error covariances in the denominator on the right side of equation (1) can be set to zero: $e_{23}=e_{14}=0$. Further, we distribute the remaining error covariance symmetrically between the remaining variables: $e_{13}=e_{24}=1-\sqrt{\frac{R_{23} R_{14}}{R_{13} R_{24}}} \approx 0.04$. This means we assume no common errors between MOZART lights and GOME Richter as well as between MOZART EDGAR and GOME Leue, but a small common error fraction of $4 \%$ between MOZART EDGAR and GOME Richter as well as MOZART lights and GOME Leue. This is a purely statistical argument, we will not try to interpret it physically. This assumption is consistent with the data, but in that case there is one degree of freedom left. For given (assumed) $e_{13}, e_{14}, e_{23}, e_{24}$, the remaining variables $e_{12}, e_{34}, e_{11}, e_{22}, e_{33}$ and $e_{44}$ can vary within ranges given by the constraint that all of them are relative errors and so must simultaneously lie between zero and one:

$$
0 \leq e_{i j} \leq 1 \quad \forall i, j
$$

the ranges are listed in Table 2.

[24] If for one of the quantities a value in the allowed range is chosen, then all the others are fixed. Limit 1 is the limit of vanishing error covariance between the model calculations $\left(e_{12}=0\right)$ and thus minimal errors for the MOZART fields and maximal errors for the GOME fields. Limit 2 is the result of vanishing error covariance between the satellite retrievals and thus minimal errors of the GOME fields (the pattern error of GOME Richter vanishes here as well) and maximal errors of the MOZART fields. Varying any of the variables beyond limit 1 would result in a negative value for $e_{12}$, and beyond limit 2 in negative values for $e_{33}$ and $e_{34}$. The range of the pattern error of GOME Richter is the only one including zero: For this data set we cannot exclude that its variance contains no error at all.

[25] These error ranges will now approximatively be compared to the error estimates given in the actual GOME retrievals, using equation (A7). For this, the additional assumption of normally distributed errors is necessary, see Appendix A2. Richter and Burrows [2002] state that their tropospheric columns could be up to a factor of two smaller than the true columns, which corresponds to $b=0 . \ldots 1 / 2$ and $c=0$ in equation (A7). Leue et al. [2001] estimate an error range of $25 \%$ to $50 \%$, corresponding to $b=1 / 4 \ldots 1 / 2$ and again $c=0$. Inserting these values together with the 
Table 2. Ranges for the Pattern Errors $e_{i i}$ (Relative Error Variances) of the Fields $X_{i}$ and Corresponding Ranges for the Weighting Factors $w_{i}$ for Standardized Fields ${ }^{\mathrm{a}}$

\begin{tabular}{lccccc}
\hline & Limit 1 & Limit 2 & & Limit 1 & Limit 2 \\
\cline { 2 - 3 } \multicolumn{1}{c}{ Field $X_{i}$} & \multicolumn{2}{c}{ Pattern Error $e_{i i}$} & & \multicolumn{2}{c}{ Weight $w_{i}$} \\
\hline MOZART EDGAR & 0.17 & 0.54 & & 0.40 & 0.00 \\
MOZART lights & 0.15 & 0.53 & & 0.49 & 0.00 \\
GOME Richter & 0.44 & 0.00 & & 0.12 & 1.00 \\
GOME Leue & 0.60 & 0.28 & & -0.02 & 0.00 \\
\hline
\end{tabular}

${ }^{\text {a Limit }} 1$ corresponds to $e_{12}=0$ and $e_{34}=0.44$; limit 2 corresponds to $e_{34}=0$ and $e_{12}=0.45$.

mean values $\bar{X}_{i}$ and standard deviations $\sigma_{i}$ of the fields in the area of interest (Table 3) into equation (A7), yields $e_{\text {Richter,app }}=0.0 \ldots 0.5$ and $e_{\text {Leue,app }}=0.3 \ldots 1.0$. These error estimates based on the retrieval itself agree very well with the independently gained error ranges from correlation analysis in Table 2; they seem to be estimated rather conservatively as it is often done if errors can only be estimated roughly.

\subsubsection{Weighting Factors: Ranges}

[26] As described in Appendix A3, for fields with known pattern errors, a combination field with minimized pattern error can be constructed, with weighting factors $w_{i}$ according to equation (A9). The error ranges of the fields correspond to ranges for the weighting factors, each allowed combination of $e_{i i}$ and $e_{i j}$ results in a unique set of $w_{i}$. While pattern errors and the fractions of the variances of the original fields in the combination are independent of constant scaling factors, this does not apply to the weighting factors: They are inversely proportional to scaling factors of the fields. Since neither mean values nor standard deviations of the fields seem to be directly comparable (see Table 3), for easier comparison, the fields were standardized before calculating the ranges for the weighting factors $w_{i}$. The limits of the ranges of the $w_{i}$ corresponding to the limits of the ranges of the $e_{i i}$ are shown in Table 2. At limit 1 (no error covariance between the two model calculations), MOZART EDGAR and MOZART lights contribute strongest to the combination field, followed by GOME Richter, whereas GOME Leue does not contribute. At limit 2 (maximal error covariance between the model calculations), the pattern error of GOME Richter vanishes: A field that is completely true would only get worse by mixing with other fields, so here the combination field solely consists of GOME Richter.

\section{Anthropogenic $\mathrm{NO}_{\mathrm{x}}$ Emissions}

[27] After examining tropospheric $\mathrm{NO}_{2}$ column densities, a correlation analysis shall now be applied to anthropogenic $\mathrm{NO}_{\mathrm{x}}$ emissions. To do this, at first emission fields must be estimated from the satellite fields, then the pattern errors of the emission fields can be determined in the area dominated by anthropogenic emissions (see section 2.3.1). Finally, a combined emission field with minimal pattern error will be constructed. By a discussion of outliers, areas will be identified where the disagreement of the emission fields and thus the uncertainty of the combination field is highest.

\subsection{Estimating Emissions From Satellite Columns}

[28] In this section, several transformations will be introduced to estimate emission fields from the GOME measurements. These transformations shall be simple, and they shall restrict the change of the distribution pattern of the GOME fields to a sharpening of the original patterns without imprinting model errors. An inversion of the model would violate both requirements, the model will be used here only to find transformations that improve the correlation of model sources and columns.

[29] Due to the short lifetime of $\mathrm{NO}_{\mathrm{x}}(\approx 1$ day), in the spatial resolution used here (T63) the distribution patterns of the emissions already closely correspond to those of the tropospheric $\mathrm{NO}_{2}$ column densities. Within the MOZART model this can be quantified by the spatial correlation between model sources and columns: Its value for the annual mean fields of emissions and resulting tropospheric columns is $R=0.82$. This leaves $1-R^{2} \approx 32 \%$ unexplained variance as a consequence of advection and turbulent diffusion as well as a spatial dependency of the lifetime and the NO fraction of the $\mathrm{NO}_{\mathrm{x}}$. For the area dominated by anthropogenic emissions (section 2.3.1), the correlation coefficient between anthropogenic emissions and column densities is nearly the same $(R=0.83)$. At least within the model, the all other emissions in that area (biomass burning, agriculture, soil emissions, and air planes and lightning as volume sources) do not change the pattern but rather have, together with a background concentration, the effect of an offset; while their fraction of the source strength in that area amounts to $17 \%$, they contribute only $1 \%$ to the spatial variance.

\subsubsection{Lifetime, NO Fraction and Source Strength:} Scaling

[30] To construct a new source not only as a pattern, but which can be used as input field for a CTM, we have to assume a realistic source strength. Since a global scaling factor does not influence the correlation coefficients, this information cannot be gained by the pattern errors. For simplicity, we will assume the strength of the anthropogenic emissions of EDGAR 3 in the area dominated by anthropogenic emissions to be correct, and scale the GOME fields accordingly.

[31] Other scaling possibilities are to account for lifetime and $\mathrm{NO}$ fraction of $\mathrm{NO}_{\mathrm{x}}$ by multiplying the column densities either as a whole field with the global model ratio of the annual mean of $\mathrm{NO}_{\mathrm{x}}$ sources to corresponding tropospheric $\mathrm{NO}_{2}$ columns (done by Leue et al. [2001]), or grid point for grid point with the corresponding local value (done by Martin et al. [2003]). Similar to the differences in the mean values in Table 3, the total source strengths of the resulting fields will differ, and scaling by local factors gained from the model changes the patterns of the satellite

Table 3. Mean Values $\bar{X}_{i}$ and Standard Deviation $\sigma_{i}$ of EDGAR 3 Anthropogenic and of the Annual Mean Tropospheric Column Density Fields $X_{i}$

\begin{tabular}{lll}
\hline \multicolumn{1}{c}{ Field $X_{i}$} & \multicolumn{1}{c}{$\bar{X}_{i}$} & \multicolumn{1}{c}{$\sigma_{i}$} \\
\hline EDGAR 3 anthropogenic & $9.50 \times 10^{10} \mathrm{~cm}^{-2} \mathrm{~s}^{-1}$ & $6.12 \times 10^{10} \mathrm{~cm}^{-2} \mathrm{~s}^{-1}$ \\
MOZART EDGAR & $2.35 \times 10^{15} \mathrm{~cm}^{-2}$ & $2.33 \times 10^{15} \mathrm{~cm}^{-2}$ \\
MOZART lights & $2.34 \times 10^{15} \mathrm{~cm}^{-2}$ & $2.04 \times 10^{15} \mathrm{~cm}^{-2}$ \\
GOME Richter & $1.17 \times 10^{15} \mathrm{~cm}^{-2}$ & $0.92 \times 10^{15} \mathrm{~cm}^{-2}$ \\
GOME Leue & $1.88 \times 10^{15} \mathrm{~cm}^{-2}$ & $0.93 \times 10^{15} \mathrm{~cm}^{-2}$ \\
\hline
\end{tabular}



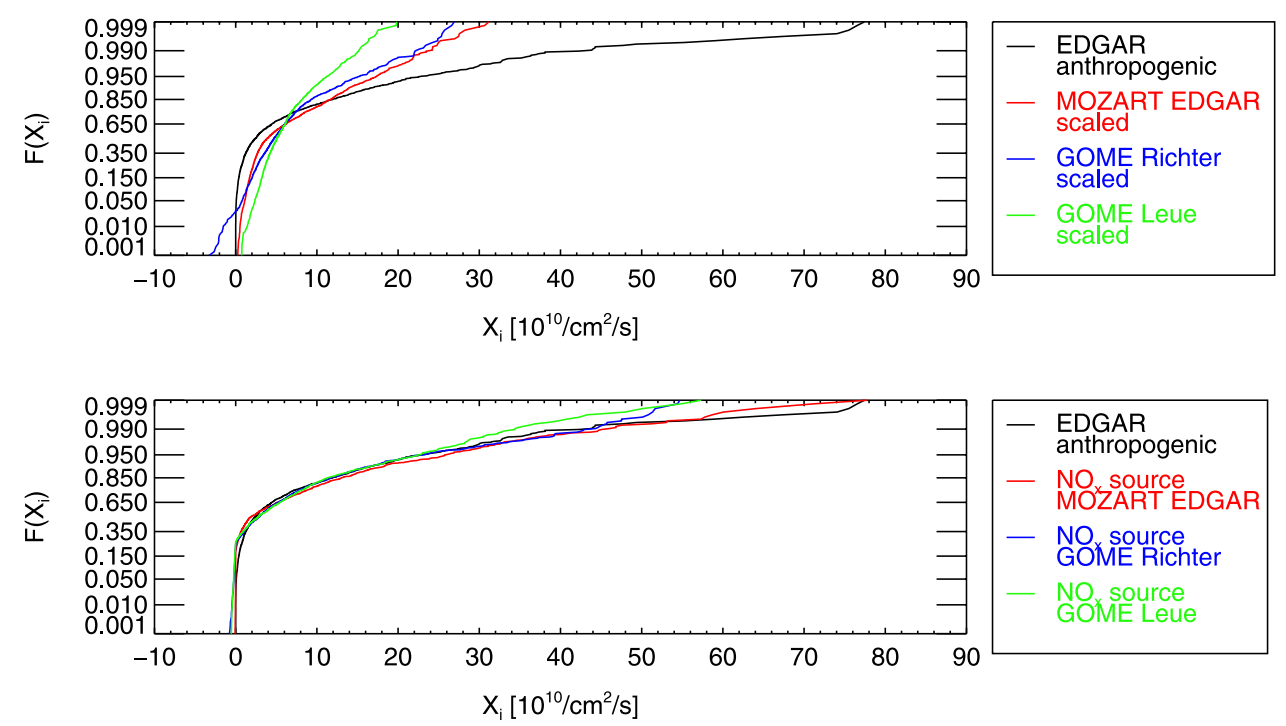

Figure 2. Cumulative frequency distribution. (top) Annual mean tropospheric column density fields of MOZART EDGAR, GOME Richter, and GOME Leue scaled to the source strength of the anthropogenic EDGAR emissions. (bottom) Annual mean of the anthropogenic emissions based on the same fields estimated by applying the transformations described in subsections 3.1.1 to 3.1.4, both in comparison with the EDGAR 3 anthropogenic emissions.

fields and is highly dependent both on model chemistry and transport, even if the model meteorology field and satellite measurements are of the same year. We therefore chose the simple scaling described above.

[32] The global scaling, however, is not sufficient to convert columns into sources: Figure 2 (top) shows the cumulative frequency distributions $F\left(X_{i}\right)$ of the annual mean EDGAR 3 anthropogenic emissions over the analyzed area, of the corresponding MOZART calculation and of the GOME tropospheric columns all scaled to the same source strength over that area. Despite the scaling, the distributions differ drastically, especially the high source values are not obtained in any of the column fields. So further transformations are needed. The model calculation will serve as a testcase, because for the model columns the corresponding anthropogenic emissions are known. The aims for the transformed columns are as follows: (1) better reproduction of the high values of EDGAR anthropogenic (better agreement of the black and red curve in Figure 2 (top)), (2) agreement with both the source strength and the variance of EDGAR anthropogenic, and (3) high correlation with EDGAR anthropogenic. The following transformations to account for turbulent diffusion and all those emissions not in our focus were developed to achieve these aims and still be simple.

\subsubsection{Turbulent Diffusion: Deconvolution}

[33] Within the lifetime of one day, most of the model transport goes to the neighboring grid points. An easy possibility to simulate turbulent diffusion to the nearest neighbors without the need of explicit assumptions about meteorological parameters is a convolution with a kernel

$$
K=\left(\begin{array}{lll}
1 & 1 & 1 \\
1 & n & 1 \\
1 & 1 & 1
\end{array}\right),
$$

which contains a parameter $n$ : The smaller $n$ the stronger the smoothing. This parameter was chosen to fulfill two criteria: To maximize the correlation of convoluted sources and corresponding model columns and simultaneously to minimize the pattern error (see section 3.2) of the satellite-based emission estimates gained by deconvoluting the GOME fields with the same kernel. This combination was achieved optimally for $n=8$. The spatial correlation coefficient between deconvoluted annual mean tropospheric column densities and corresponding source fields increased to $R=0.90$.

\subsubsection{Other Emissions and Background Concentration: Offsets}

[34] As explained above, emissions from biomass burning, agriculture, soil, air planes and lightning as well as background concentrations have the effect of an offset even in the model fields. The satellite fields may have additional offsets of positive or negative signs resulting from the subtraction of the stratospheric columns in the retrieval. Similar to scaling factors, offsets have no influence on correlation coefficients or pattern errors but have to be eliminated to obtain a realistic emission field. It is assumed here that the EDGAR 3 anthropogenic emissions don't have an offset; the offsets of the other fields are identified relative to that field.

[35] To identify offsets, we focused on the small values in the cumulated frequency distribution $F\left(X_{i}\right)$. In Figure 2 (top), the scale of the $y$-axis is chosen so that a Gaussian distribution would be displayed as a straight line. For small values, the slope of $F\left(X_{i}\right)$ of the scaled GOME fields is nearly constant, which may be interpreted as Gaussian noise, both instrumental and due to transport. After deconvolution, this noise is even amplified. To eliminate the offsets relative to EDGAR 3 anthropogenic, noise was added to that source, which decreased the slope of the corresponding $F\left(X_{i}\right)$ and shifted the beginning of that curve 

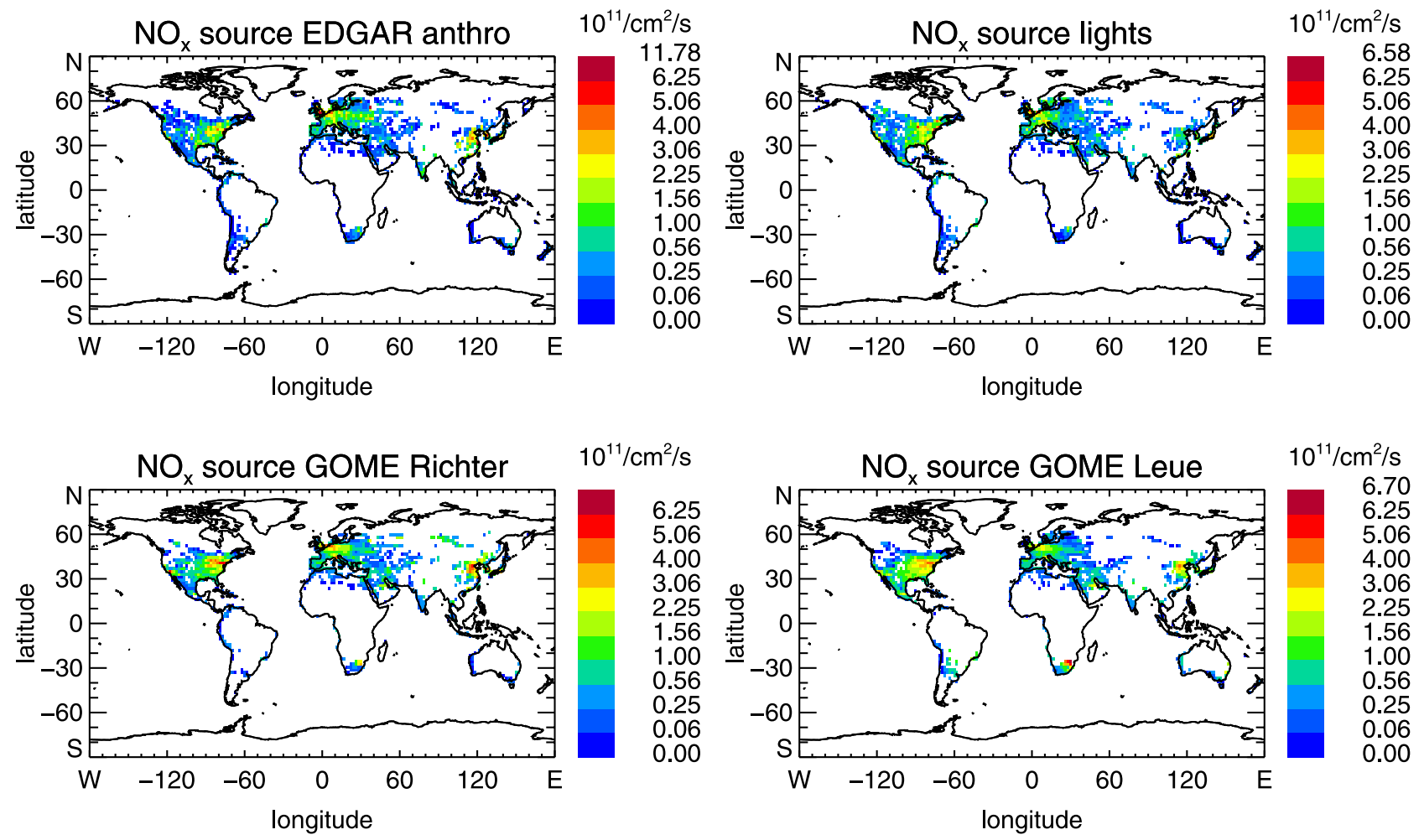

Figure 3. Annual mean values for the estimates of anthropogenic $\mathrm{NO}_{\mathrm{x}}$ emission fields: EDGAR 3 anthropogenic, light-based, GOME-based (2 retrievals), color scale: logarithmic.

to negative values. Then an offset was subtracted from the other fields followed by rescaling to the EDGAR source strength. Both were done iteratively until, for small values, the curves of $F\left(X_{i}\right)$ for source and former column fields agreed well.

\subsubsection{Maxima and Variance: Exponent}

[36] After deconvolution, elimination of offsets and rescaling, the model-based source estimate $X_{\text {col, est }}$ still does not reproduce the maxima and the variance of the corresponding anthropogenic emissions $X_{s r c}$. The relationship between the EDGAR-based model columns and the corresponding sources is slightly nonlinear: A linear fit of logarithmized columns and sources performed using the algorithm fitexy described in the numerical recipes [Press et al., 1997] assuming errors in both coordinates yields a slope of $a_{E D}=1.16$, which for the not-logarithmized fields corresponds to an exponent $X_{s r c}=X_{c o l}^{a}$, est. Since this exponent is smaller for the lights $\left(a_{l i}=1.05\right)$, in the following, the positive values of the column fields were potentiated with the mean value $a_{m}=1.11$, followed by a rescaling.

[37] By the exponent, the high values are increased more than the low values. By the rescaling, all values were decreased equally, so as a net effect, small values were decreased and high values were increased which suppresses the noise at the origin of the curves of the cumulative frequency distributions. In Figure 2 at the bottom, the curves $F\left(X_{i}\right)$ are shown both for the EDGAR anthropogenic emissions and for the column-based source estimates after rescaling, deconvolution, elimination of offsets and potentiation. These curves agree quite well.
[38] After the potentiation, the correlation between the annual mean of the EDGAR 3 anthropogenic source and the corresponding column-based source estimate is again slightly increased to $R=0.92$. The unexplained variance between model columns and corresponding anthropogenic sources in the examined area could altogether be reduced by all transformations from $32 \%$ (see section 3.1 ) to $1-R^{2} \approx$ $15 \%$. Considering the fact that the spatial dependency of the lifetime and of the NO as well as the distribution pattern of the other emissions are unaccounted for and that transport is only partly considered by deconvolution and potentiation, this reduction is remarkable. A reduction beyond that would require increasingly sophisticated and potentially erroneous assumptions where there is little potential for improvement left.

[39] In Figure 3 in the lower two panels, the GOMEbased anthropogenic $\mathrm{NO}_{\mathrm{x}}$ emission estimates in the area dominated by anthropogenic emissions are shown with a logarithmic color scale. In comparison to the original

Table 4. Spatial Correlation Coefficients $R_{i j}$ of the Annual Mean Source Field Estimations and Untransformed GOME Retrievals in the Analyzed Area ${ }^{a}$

\begin{tabular}{lccccc}
\hline & & & & GOME & GOME \\
$R_{i j}$ & $X_{l i}$ & $X_{R i}$ & $X_{\text {Leu }}$ & Richter & Leue \\
\hline$X_{E D}$ & $0.73 \pm 0.03$ & $0.69 \pm 0.04$ & $0.57 \pm 0.04$ & $0.67 \pm 0.04$ & $0.55 \pm 0.04$ \\
$X_{l i}$ & & $0.66 \pm 0.04$ & $0.59 \pm 0.04$ & $0.64 \pm 0.04$ & $0.57 \pm 0.04$ \\
$X_{R i}$ & & & $0.83 \pm 0.02$ & $0.93 \pm 0.02$ & \\
$X_{\text {Leu }}$ & & & & & $0.96 \pm 0.02$ \\
\hline
\end{tabular}

${ }^{\mathrm{a}}$ Error estimates: one standard deviation, gained by the bootstrap method. 
Table 5. Relative Error Variances $e_{i i}$ and Corresponding Weighting Factors $w_{i}$ for the Optimal Combination of the Fields $X_{i}$ and Spatial Correlation Coefficients Between the Four Input Source Fields and the Combination Source in the Area Dominated by Anthropogenic Emissions ${ }^{\mathrm{a}}$

\begin{tabular}{lcccc}
\hline & $X_{E D}$ & $X_{l i}$ & $X_{R i}$ & $X_{\text {Leu }}$ \\
\hline$e_{i i}$ & $0.27 \pm 0.05$ & $0.28 \pm 0.06$ & $0.40 \pm 0.05$ & $0.56 \pm 0.06$ \\
$w_{i}$ & $0.34 \pm 0.07$ & $0.45 \pm 0.08$ & $0.19 \pm 0.04$ & $0.02 \pm 0.03$ \\
$R_{X_{i}, X_{c}}$ & $0.92 \pm 0.01$ & $0.92 \pm 0.01$ & $0.82 \pm 0.02$ & $0.71 \pm 0.03$ \\
\hline
\end{tabular}

${ }^{\text {a }}$ Error estimates: one standard deviation, gained by the bootstrap method; relative error covariance of the two GOME fields: $e_{34}=0.38 \pm 0.05$.

column density fields in Figure 1 (linear color scale), the distribution pattern is sharpened. In Table 4, the correlation coefficients of the anthropogenic source estimates (EDGAR and lights) and the GOME columns with and without these transformations are listed.

\subsection{Pattern Errors}

[40] For the four anthropogenic emission fields depicted in Figure 3, pattern errors can be calculated, since the errors of three fields, EDGAR $\left(X_{E d}\right)$, light-based $\left(X_{l i}\right)$ and GOMEbased $\left(X_{R i}, X_{L e u}\right)$, can be assumed to be independent. For these errors there will be given uncertainty estimates. These estimates were done with the bootstrap method and correspond to one standard deviation.

[41] The pattern errors for the four emission fields can be calculated by equation (A5), using the correlation coefficients listed in Table 4. As for the column density fields, the equation is a system of six equations and ten unknown variables. As for the columns, the common errors of the source estimates $X_{E D}$ and $X_{l i}$ with the GOME-based estimates $X_{R i}$ and $X_{L e u}$, respectively, $\left(e_{13}, e_{14}, e_{23}, e_{24}\right)$ will be assumed to be as small as possible. The minimal values for these quantities compatible with the correlation coefficients in Table 4 follow, analogous to the columns, from the relation $e_{23}=e_{14}=0$ and $e_{13}=e_{24}=1-\sqrt{\frac{R_{23} R_{14}}{R_{13} R_{24}}} \approx$ $0.05 \pm 0.02$. Contrary to the column fields, there is no model applied here to both EDGAR anthropogenic and the light source. So here it is possible to further assume no common pattern errors between EDGAR anthropogenic and the lightbased $\mathrm{NO}_{\mathrm{x}}$ source: $e_{12}=0$. With these assumptions, equation (A5) can be solved, the solutions are listed in Table 5 (top).

[42] As explained in section A2, a pattern error cannot be transformed into a mean error at each grid point. But assuming the error model equation (A6) to hold for $X_{E D}$, we can calculate parameters consistent with the pattern error $e_{E D}=0.27 \pm 0.05$ found here. Solving equation (A6) for $b$ with $c=0$ (no offset) and inserting the values for $\bar{X}_{E D}$ and $\sigma X_{E D}$ from Table 3, we obtain $b \approx 0.5$. This estimate for the mean error at each grid point of EDGAR anthropogenic (fossil, industry, waste, see above) is twice as big as the stated error for its total ( $25 \%$ at one standard deviation).

\subsection{Construction of a Combined Emission Field}

[43] Once the pattern errors of the emission fields are known, an optimized field can be constructed. This will be done first for the area the pattern errors were calculated for and then be extended to the rest of the globe.

\subsubsection{Area Dominated by Anthropogenic Emissions}

[44] As described in Appendix A in section A3, the emission fields can be combined minimizing the pattern error of the resulting field. The corresponding weighting factors are also listed in Table 5. One aim of this analysis is the construction of a new source field with improved distribution pattern of the anthropogenic $\mathrm{NO}_{\mathrm{x}}$ source, but also with realistic source strength. So here the weighting factors $w_{i}$ do not refer to standardized fields but to the fields as constructed in section 3.1. After the transformations described in that section, the fields only slightly differ in their variances, so the weighting factors for these fields and the standardized fields differ less than the stated error estimates of one standard deviation gained by the bootstrap method.

[45] The pattern error of the combined field is given by equation (A8) and amounts to $e_{c}=0.13 \pm 0.02$, which is a reduction of $50 \%$ compared to the $e_{E D}=0.27 \pm 0.05$, the smallest pattern error of the input fields.

[46] In Table 5 (bottom), the spatial correlation coefficients between the input fields and the combination are shown. Apart from the correlation between the two satellite source estimates, which contain common errors, the correlations of the input fields with the combination are higher than between the input fields themselves (compare Table 4). 3.3.2. Global Emission Field

[47] To be able to construct a global emission field, we assumed that the pattern errors of the input fields gained in the area dominated by anthropogenic emissions are also true for the rest of the globe. Using the relative error variances $e_{i i}$ and error covariances $e_{i j}$ listed in Table 5, we calculated weighting factors for the combination minimizing the pattern errors of three and two fields, respectively, analogue to the combination of four fields.

[48] A realistic emission field must not contain negative values. As the small values in the GOME-based source fields are dominated by noise, which was in part amplified by deconvolution, we applied as a minimum threshold for the satellite fields not zero but $1 \times 10^{10} \mathrm{~cm}^{-2} \mathrm{~s}{ }^{-1}$. So we combined the four input fields at those continental grid points of the area dominated by anthropogenic emissions where both GOME-based source estimates displayed defined values greater than $1 \times 10^{10} \mathrm{~cm}^{-2} \mathrm{~s}^{-1}$ (area 1). At the continental anthropogenically dominated grid points where either $X_{L e u}$ or $X_{R i}$ was below the threshold, only $X_{E D}, X_{l i}$ and

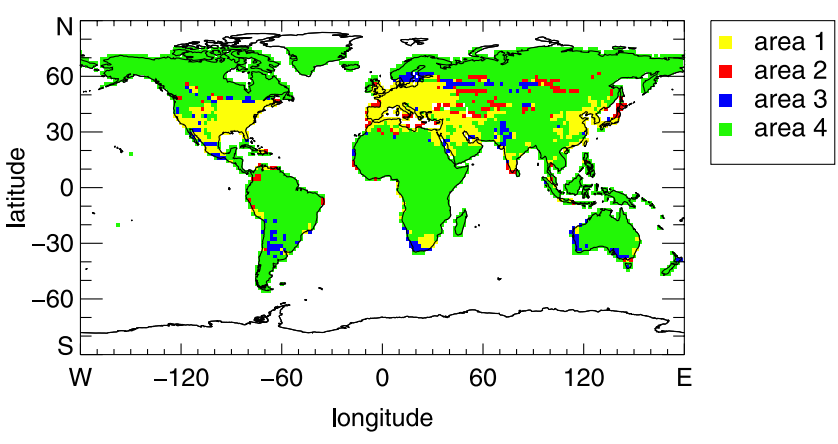

Figure 4. Area 1: $X_{E D}, X_{l i}, X_{R i}$ and $X_{L e u}$ will be combined; area 2: $X_{E D}, X_{l i}$ and $X_{R i}$, area 3: $X_{E D}, X_{l i}$ and $X_{L e u}$, area 4: $X_{E D}$ and $X_{l i}$ will be combined, respectively. 
Table 6. Weighting Factors Resulting From the Error Variances in Table 5 to Minimize the Pattern Error in the Combination of Four, Three, and Two Fields, Number of Grid Points in the Corresponding Area Over Land, and Resulting Pattern Error of the Combination

\begin{tabular}{|c|c|c|c|c|c|c|}
\hline Area & $w_{E D}$ & $w_{l i}$ & $w_{R i}$ & $w_{\text {Leu }}$ & $M$ & $e_{c}$ \\
\hline 1 & $0.34 \pm 0.07$ & $0.45 \pm 0.08$ & $0.19 \pm 0.04$ & $0.02 \pm 0.03$ & 789 & $0.13 \pm 0.02$ \\
\hline 2 & $0.34 \pm 0.05$ & $0.46 \pm 0.07$ & $0.20 \pm 0.04$ & - & 160 & $0.13 \pm 0.02$ \\
\hline 3 & $0.38 \pm 0.06$ & $0.46 \pm 0.07$ & - & $0.16 \pm 0.04$ & 198 & $0.14 \pm 0.02$ \\
\hline 4 & $0.44 \pm 0.06$ & $0.56 \pm 0.07$ & 一 & - & 3712 & $0.16 \pm 0.03$ \\
\hline
\end{tabular}

either $X_{R i}$ or $X_{L e u}$ were combined (area 2 and area 3, respectively). At those continental grid points that were either dominated by other emissions or where both GOMEbased sources were below the threshold or undefined, only $X_{E D}$ and $X_{l i}$ were combined (area 4). The four areas with differing combinations of the fields are shown in Figure 4. Over the oceans, the ship emissions were taken from EDGAR 3. The values of the weighting factors $w_{i}$ in each area, the number of grid points $M$ where this combination was built and the resulting pattern error $e_{c}$ of that combination are listed in Table 6. To obtain a global field, we put those areas together and built the mean value of the single pattern errors weighted with the corresponding number of grid points. This results in a total pattern error for continental anthropogenic $\mathrm{NO}_{\mathrm{x}}$ emissions of $e_{c \text {, tot }}=0.15 \pm 0.03$. The four input fields and the resulting combination in the area dominated by anthropogenic emissions are shown enlarged for North America, Europe and East Asia in Figure 5. The main difference between EDGAR 3 anthropogenic and the combination source is that in the latter the industrial hot spots are not as pronounced compared to other cities and less populated areas as in EDGAR.

\subsection{Outliers: Areas That Need Further Investigation}

[49] Correlation coefficients, error variances and weighting factors are properties of the total fields $X_{i}$, they cannot be attributed to single grid points. But single large outliers in the fields can reduce correlations and by that increase pattern errors significantly. By their identification, areas will be determined where the uncertainty of the combination field is highest and which therefore need further investigation. The outliers were identified as described in Appendix A in section A4 to the $\alpha=0.005$ level of significance.

[50] Since all of the outliers are found either in North America, Europe, East Asia or South Africa, their geographical location in these areas is shown enlarged in Figure 6. The most significant outliers are those of the light source in eastern China (source strength of the light emissions far too low, in agreement with Doll et al. [2000], see section 2.2.2) and those of the source estimation based on GOME Leue over the Highveld near Johannesburg in South Africa (GOME Leue too high). In eastern China, outliers are identified in other fields as well: GOME Richter and EDGAR both have higher values there than the lights, but the patterns do not coincide. China is an area of rapid growth over time [see, e.g., Richter et al., 2005], where a combination of fields for the year 1995 (EDGAR 3, lights) and mean fields for the years 1996-2001 (GOME) is bound to be error prone, so that further investigations are necessary.

[51] Many outliers of the EDGAR anthropogenic source are located at cities with high emissions (e.g. New York or
London). This could be due to EDGAR overestimating industrial hot spots in the northern hemisphere. But since transport plays an important role at these cities (high gradient of the emissions, advection of clean air) it is also possible that at those locations the transformations applied to the GOME columns were insufficient to compensate for transport, so that their true values at these places would actually be higher. The grid point containing London for example is situated (at resolution T63) close enough to the Atlantic Ocean to get advection of clean air by mostly westerly winds, which we could not compensate for at all. In addition to that, at grid points where two fields display outliers, those in one field could be induced by outliers in the other field; therefore it is not possible to determine uniquely whether only one of the fields and if so which of them, or both fields are responsible for the outlier. We therefore didn't eliminate the outliers for the combination but use them to indicate areas where the uncertainties both of the combination and of the input fields is highest.

\section{Summary and Outlook}

[52] The method of correlation analysis developed here allows the determination of relative error variances for at least three data sets whose errors can be assumed to be independent. As an example, the method could be a tool for an instrument intercomparison where several independent instruments are measuring time series of the same physical quantity. In this paper, the method was applied to annual mean tropospheric $\mathrm{NO}_{2}$ column density fields over areas dominated by anthropogenic emissions measured by the GOME satellite instrument and modeled by the global CTM MOZART-2 as well as to anthropogenic $\mathrm{NO}_{\mathrm{x}}$ emission fields. The emissions used were the EDGAR 3 inventory and an estimation for anthropogenic $\mathrm{NO}_{\mathrm{x}}$ emissions based on satellite images of human settlements during night time. To compare the columns, the MOZART model was applied to the emission fields, to compare the emissions, simple transformations were developed to transform the GOME columns into emission estimates. Assuming independent errors for the three data sources GOME, EDGAR and lights, ranges could be given for the pattern errors of the $\mathrm{NO}_{2}$ column density fields, and pattern errors with stated uncertainties could be determined for the $\mathrm{NO}_{\mathrm{x}}$ emission fields. The pattern error of the EDGAR 3 anthropogenic $\mathrm{NO}_{\mathrm{x}}$ emission field obtained was $(27 \pm 5) \%$, which could be reduced by combining all fields to $(15 \pm 3) \%$.

[53] In those areas indicated by the outliers, further analyses are required. When available, long time series of 

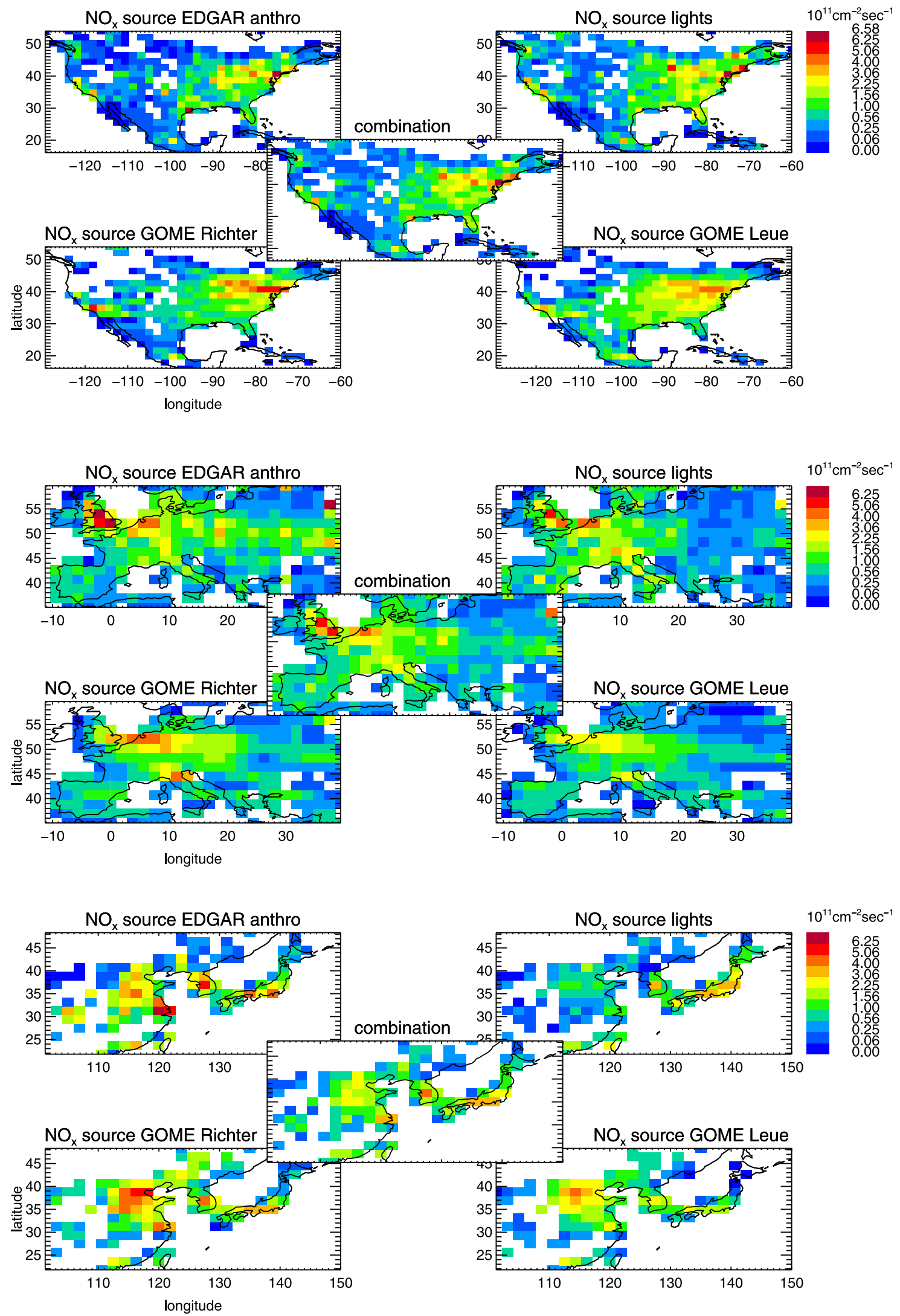

Figure 5. Areas dominated by anthropogenic emissions: input source fields and optimal combination. (top) North America, (middle) Europe, and (bottom) East Asia. 

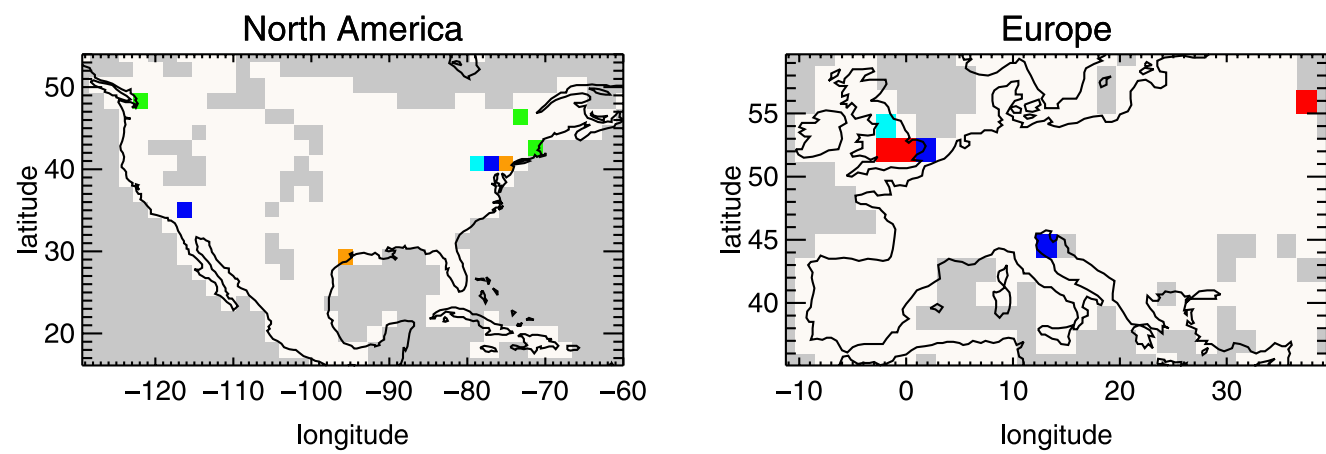

East Asia

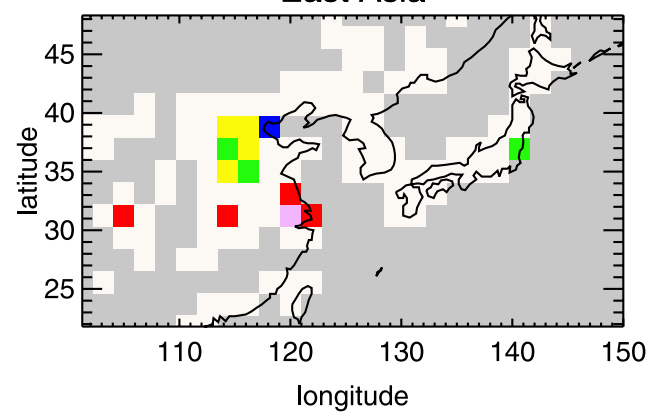

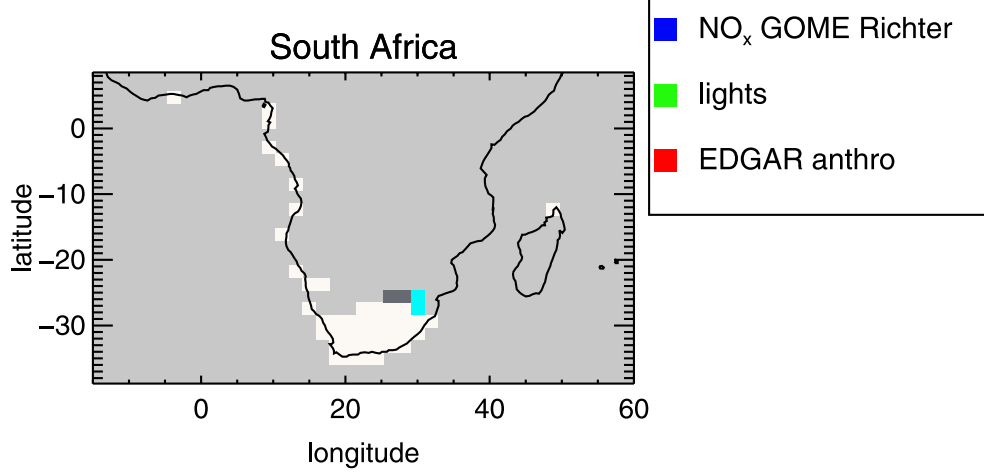

Figure 6. Geographical localization of the outliers: colored: grid points identified as outliers for one or two fields, white: outliers for none of the fields, light grey: grid points outside the analyzed area (mask).

other satellite instruments with higher precision and spatial resolution, as e.g. SCIAMACHY on ENVISAT, should reduce the uncertainties. Such data will also allow an analysis at region level, which is beyond the scope of this study, since it requires a higher number of grid points per region for error statistics and the consideration of seasonal variability and the correct meteorology.

\section{Appendix A: Correlation Analysis}

[54] The relationship between correlation coefficients and error variances and covariances is derived; it will be shown how fields with known errors can be combined to minimize the relative error variance of the combination, and the treatment of outliers in this paper is explained.

\section{A1. Correlation Coefficients and Pattern Errors}

[55] The square of the linear correlation coefficient $R_{i j}$ [Hartung and Elpelt, 1987] of two quantities $X_{i}$ and $X_{j}$

$$
R_{i j}^{2}=\frac{\operatorname{cov}\left(X_{i}, X_{j}\right)^{2}}{\operatorname{var}\left(X_{i}\right) \operatorname{var}\left(X_{j}\right)},
$$

can be interpreted as the fraction of common variance between the two. Here, we assume the examined fields to be proxies for an unknown true field $X_{\text {true }}$ and therefore express each as a linear combination of $X_{\text {true }}$ and an error field $\eta$ :

$$
X_{i}=a_{i} X_{\text {true }}+\eta_{i}, \quad X_{j}=a_{j} X_{\text {true }}+\eta_{j} .
$$

[56] Without loss of generality one can assume the error fields to be uncorrelated to the true field:

$$
\operatorname{cov}\left(X_{\text {true }}, \eta_{k}\right)=0, \quad k=i, j
$$

because if there is a correlation, one can always split up $\eta_{k}=$ $\eta_{k, \|}+\eta_{k, \perp}$ into one part $\eta_{k, \|}$ correlated to $X_{\text {true }}$ with $R=1$ and one part $\eta_{k, \perp}$ uncorrelated to $X_{\text {true }}$. In equation (A2), $\eta_{k, \|}$ can be drawn into the first part of the sum changing the coefficient of the true field. For the remaining $\eta_{k, \perp}$, equation (A3) is valid. Using the definitions

$$
e_{i i}:=\frac{\operatorname{var}\left(\eta_{i}\right)}{\operatorname{var}\left(X_{i}\right)}, \quad e_{i j}:=\frac{\operatorname{cov}\left(\eta_{i}, \eta_{j}\right)}{\operatorname{cov}\left(X_{i}, X_{j}\right)}
$$

for the relative error variance $e_{i i}$ and the relative error covariance $e_{i j}$, inserting the linear combination (A2) into the formula for the linear correlation coefficient (A1) and using equation (A3) yields after some transformations

$$
R_{i j}^{2}\left(1-e_{i j}\right)^{2}=\left(1-e_{i i}\right)\left(1-e_{j j}\right) .
$$

This equation connects the relative error variances and covariances of the examined fields and their linear correlation coefficients. As the correlation coefficients, the error variances and covariances do not depend on a constant factor or a constant offset of one of the fields; they don't refer to absolute values but only to the patterns of the fields. If spatial correlations between two dimensional fields are examined, the relative error variances $e_{i i}$ will be also called pattern errors. 
[57] For $N$ fields, equation (A5) is a system of $\left(\begin{array}{c}N \\ 2\end{array}\right)$ equations with $\left(\begin{array}{c}N \\ 2\end{array}\right)+N$ unknown variables, so in general, the system is not solvable. Only if there is already external information about some of the variables, for instance if one can safely assume the errors of the examined fields to be independent $\left(e_{i j}=0\right)$, one can solve the system for the rest of the variables. If there are at least three fields with independent errors, equation (A5) is solvable.

\section{A2. Pattern Error and Mean Error at Each Grid Point}

[58] A pattern error characterizes a field as a whole, while an error field relates to the value of the corresponding field at each grid point, often given as percentage. If the functional dependency between errors and true values is known, the pattern error can be calculated from the percentage errors. The other direction is not possible since the fields in equation (A2) are only defined up to a common factor which would change the absolute error but not the pattern error.

[59] Errors of measurements often consist of a part $\eta_{1}=$ $b X_{\text {true }} \xi_{1}$ proportional to the true value and a noisy part $\eta_{2}=$ $c \xi_{2}$ according to the limit of detection, where $\xi_{1}$ and $\xi_{2}$ are noisy variables uncorrelated to each other and the true field. Stated are the values $b=\operatorname{stddev}\left(\eta_{1} / X_{\text {true }}\right)$ and $c=\operatorname{stddev}\left(\eta_{2}\right)$ of the error model

$$
\begin{gathered}
X=X_{\text {true }}+\eta_{1}+\eta_{2}, \\
\eta_{1}=b X_{\text {true }} \xi_{1}, \quad \eta_{2}=c \xi_{2}, \quad \text { with } \\
\left\langle\xi_{i}\right\rangle=0,\left\langle\xi_{i} \xi_{j}\right\rangle=\delta_{i j},\left\langle X_{\text {true }} \xi_{i}\right\rangle=0, \quad i, j=1,2 .
\end{gathered}
$$

This error shall now approximatively be transformed into the pattern error in equation (A4) resulting from the error model in equation (A2), where no functional form of $\eta$ was assumed. For this, one additional assumption has to be made: $\xi_{i}$ shall be normally distributed. This is only approximatively true. With this assumption, uncorrelated variables are also statistically independent, and the expected value of a product of independent variables equals the product of their expectation values. Using this, $X_{\text {true }}$ and $\eta_{1}$ must be uncorrelated,

$$
\left\langle X_{\text {true }} \eta_{1}\right\rangle=b\left\langle X_{\text {true }}^{2} \xi_{1}\right\rangle=b\left\langle X_{\text {true }}^{2}\right\rangle\left\langle\xi_{1}\right\rangle=0,
$$

so with this assumption, the coefficient $a$ in the error model in equation (A2) can be set to one, since there is no need to compensate for a correlation between $X_{\text {true }}$ and $\eta$ and there is no coefficient of $X_{\text {true }}$ in equation (A6). The approximation for the pattern error resulting from the error model in equation (A6) reads as

$$
e_{X, a p p}=\frac{\operatorname{var}\left(\eta_{1}\right)+\operatorname{var}\left(\eta_{2}\right)}{\operatorname{var}(X)}
$$

which can be transformed into

$$
e_{X, a p p}=\frac{\frac{b^{2}}{1+b^{2}}\left(\operatorname{var}(X)+\bar{X}^{2}-c^{2}\right)+c^{2}}{\operatorname{var}(X)}
$$

using the error model of equation (A6), the definition of the variance and the assumption of normally distributed errors.

\section{A3. Construction of a Combination Field}

[60] To construct an optimized combination field, first a transformation has to be carried out to get fields with independent errors. For these fields, weighting factors can be calculated to minimize the relative error variance of the combination field. These weighting factors can than be transformed back.

\section{A3.1. Fields With Independent Errors}

[61] If equation (A5) is solvable, the relative error variances and covariances of the involved fields can be calculated from the correlation coefficients. They can be used to combine the fields to obtain a new field with minimized relative error covariance. To avoid counting common errors twice in the combination, a transformation is applied to get fields with uncorrelated errors. This is done by diagonalizing the error covariance matrix $E$, which contains the absolute error variances and covariances $\left(E_{i i}=e_{i i} \cdot \operatorname{var}\left(X_{i}\right), E_{i j}=e_{i j} \cdot \operatorname{cov}\left(X_{i}, X_{j}\right)\right)$. Since $E$ is positive definite and symmetric, its eigenvalues $\lambda_{i}$ are positive and real and the normalized eigenvectors $\vec{v}_{i}$ can be used as a transformation matrix $P=\left(\vec{v}_{1}\left|\vec{v}_{2}\right| \mid \vec{v}_{N}\right)$. This transformation yields the diagonalized error covariance matrix $D$ and fields with uncorrelated errors $Y_{i}\left(\left(X_{1}, X_{2}, \ldots X_{N}\right)^{\top}=\vec{X}\right)$

$$
D=P^{\top} E P \text { and } \vec{Y}=\vec{P}^{\top} \vec{X}
$$

This procedure is a primary component analysis [Storch and Zwiers, 1999] for the error fields.

\section{A3.2. Weighting Factors}

[62] The transformed fields $Y_{i}$ are linear combinations of the original fields $X_{i}$, and as these they can be presented as linear combination of a true field $X_{\text {true }}$ and an error field $\xi_{i}$. Since this analysis is independent of constant offsets or factors, $X_{\text {true }}$ and $\xi_{i}$ can be standardized: $X_{\text {true }}^{\text {std }}$, the variances are accounted for by the coefficients $a_{i}$ and $b_{i}$ :

$$
\begin{gathered}
Y_{i}=a_{i} X_{\text {true }}^{\text {std }}+b_{i} \xi_{i} \quad \text { with } \\
\operatorname{cov}\left(X_{\text {true }}^{\text {std }}, \xi_{i}\right)=0, \quad \operatorname{cov}\left(\xi_{i}, \xi_{j}\right)=\delta_{i j} .
\end{gathered}
$$

As for the untransformed error fields $\eta_{i}$ in equation (A3), the covariance between the $\xi_{i}$ and the true field can be set to zero without loss of generality. After the transformation, also the covariances between different error fields are zero. The coefficients are obtained from the eigenvalues of the error covariance matrix: $b_{i}= \pm \sqrt{\lambda_{i}}$ and $a_{i}= \pm$ $\sqrt{\operatorname{var}\left(Y_{i}\right)-\lambda_{i}}$. The sign of $a_{i}$ must be chosen so that the true field is contained with the same sign in all fields $Y_{i}$ (all correlations between the $Y_{i}$ must be positive), the sign of the coefficient $b_{i}$ of the error field is arbitrary.

[63] The transformed fields $Y_{i}$ can be combined

$$
\begin{gathered}
Y_{c}=\sum_{i=1}^{N} c_{i} Y_{i}=a_{c} X_{t r u e}^{s t d}+\xi_{c}, \\
a_{c}=\sum_{i=1}^{N} c_{i} a_{i}, \quad \xi_{c}=\sum_{i=1}^{N} c_{i} b_{i} \xi_{i},
\end{gathered}
$$


with coefficients $c_{i}$ minimizing the relative error variance of the combined field:

$$
\begin{aligned}
e_{c}\left(c_{1}, \cdots, c_{N}\right) & =\frac{\operatorname{var}\left(\eta_{c}\right)}{\operatorname{var}\left(Y_{c}\right)} \\
& =\frac{\sum_{i=1}^{N} c_{i}^{2} b_{i}^{2}}{\left(\sum_{i=1}^{N} c_{i} a_{i}\right)^{2}+\sum_{i=1}^{N} c_{i}^{2} b_{i}^{2}} \\
& =\min .
\end{aligned}
$$

The minimum is found for

$$
c_{i} \propto \frac{a_{i}}{b_{i}^{2}}=\frac{\sqrt{\operatorname{var}\left(Y_{i}\right)-\lambda_{i}}}{\lambda_{i}},
$$

so the weighting factors are proportional to the standard deviation of the fraction of the true field contained in the fields and inversely proportional to the variance of the error field contained in the fields. The factors become unique by the constraint $\Sigma_{i=1}^{N} c_{i}=1$. To obtain the weighting factors $w_{i}$ for the original fields $X_{i}$, the $c_{i}$ are transformed back:

$$
\vec{w}=P \vec{c}
$$

\section{A4. Outliers}

[64] Outliers of a field $X_{i}$ are defined in this analysis as data points that contribute strongly to the error variance of that field. To identify them, the contribution of each grid point to the pattern errors of the analyzed fields is calculated.

\section{A4.1. Influence of Single Grid Points on the Pattern Errors}

[65] We calculated the error variance of each field once using all data points and once omitting each single data point in turn. This procedure is known as the jackknife [Efron, 1982]. Since the omission of one data point simultaneously changes the error variance and the variance of the field, to separate the two effects the changes are calculated for the absolute error variances $E_{i i}=e_{i i} \cdot \operatorname{var}\left(X_{i}\right)$. For easier comparison between the fields, the changes are normalized with the total error variance. For each field $X_{i}$ at every data point $l$ the quantity

$$
\Delta e_{i i}^{l}:=\frac{E_{i i}^{(l)}-E_{i i}}{E_{i i}}
$$

is determined. The notation $y^{(l)}$ represents the quantity $y$ omitting data point $l$. For the jackknife fields $\Delta e_{i i}^{l}$, data points are identified as outliers if the standardized deviation from the mean exceeds some test quantity $T_{M, \alpha}$

$$
\frac{\overline{\Delta e_{i i}}-\Delta e_{i i}^{l}}{\operatorname{stddev}\left(\Delta e_{i i}\right)}>T_{M, \alpha}
$$

where $M$ is the total number of data points and $\alpha$ is the desired level of significance. This is a one-sided test for data points that enlarge the pattern error.

\section{A4.2. Identification of Outliers}

[66] The significance level used to identify outliers in the fields $\Delta e_{i i}^{l}$ (equation (A10)) was $\alpha=0.005$. The test quantity $T_{M, \alpha}$ for the outlier test in equation (A11) was determined by Monte Carlo Simulation: The frequency distribution of the $\Delta e_{i i}^{l}$ is roughly a lognormal distribution. We chose the parameters for a lognormally distributed stochastic variable so that its frequency distribution closely resembled that of the $\Delta e_{i i}^{l}$. Then we determined $T_{M, \alpha}$ so that for $N=10000$ realizations of that stochastic variable with a samplesize of $M=1463$ containing no outliers, an average of $0.5 \%$ outliers was identified.

[67] Acknowledgments. This work was funded by the German Federal Ministry for Education and Research (BMBF) in the AFO-2000 project ISOTROP, the HGF (Helmholtz Association of National Research Centres) Networking Fund "Generation and validation of ENVISAT data products and their usage to investigate the upper troposphere and stratosphere," and is also a contribution to ACCENT TROPOSAT-2.

\section{References}

Beirle, S., U. Platt, M. Wenig, and T. Wagner (2004), Highly resolved global distribution of tropospheric $\mathrm{NO}_{2}$ using GOME narrow swath mode data, Atmos. Chem. Phys., 4, 1913-1924.

Boersma, K. F., H. J. Eskes, and E. J. Brinksma (2004), Error analysis for tropospheric $\mathrm{NO}_{2}$ retrieval from space, J. Geophys. Res., 109, D04311, doi:10.1029/2003JD003962.

Burrows, J. P., et al. (1995), The global ozone monitoring experiment (GOME): Mission concept and first scientific results, J. Atmos. Sci., $56,151-175$.

Doll, C. N. H., J.-P. Muller, and C. D. Elvidge (2000), Nighttime imagery as a tool for global mapping of socioeconomic parameters and greenhouse gas emissions, Ambio, 29(3), 157-162.

Dubach, L., and C. Ng (1988), NSSDC's compendium of meteorological space programs, satellites, and experiments, technical report, Natl. Space Sci. Data Cent., Greenbelt, Md.

Efron, B. (1982), The jackknife, the bootstrap and other resampling plans, in Regional Conference Series in Applied Mathematics, Soc. for Ind. and Appl. Math., Philadelphia, Pa.

Elvidge, C. D., M. L. Imhoff, K. E. Baugh, V. R. Hobson, I. Nelson, J. Safran, J. B. Dietz, and B. T. Tuttle (2001), Nighttime lights of the world: 1994-1995, ISPRS J. Photogram. Remote Sens., 56, 81-99.

Hartung, J., and B. Elpelt (1987), Statistik, Oldenbourg Verlag München, Wien.

Horowitz, L.W., et al. (2003), A global simulation of tropospheric ozone and related tracers: Description and evaluation of MOZART, version 2, J. Geophys. Res., 108(D24), 4784, doi:10.1029/2002JD002853.

Lauer, A., M. Dameris, A. Richter, and J. P. Burrows (2002), Tropospheric $\mathrm{NO}_{2}$-columns: A comparison between model and retrieved data from GOME measurements, Atmos. Chem. Phys., 2, 67-78.

Leue, C., M. Wenig, T. Wagner, O. Klimm, U. Platt, and B. Jähne (2001), Quantitative analysis of $\mathrm{NO}_{\mathrm{x}}$-emissions from global ozone monitoring experiment satellite image sequences, J. Geophys. Res., 106, $5493-$ 5505.

Martin, R. V., et al. (2002), An improved retrieval of tropospheric nitrogen dioxide from GOME, J. Geophys. Res., 107(D20), 4437, doi:10.1029/ 2001JD001027.

Martin, R. V., D. J. Jacob, K. Chance, T. P. Kurosu, P. I. Palmer, and M. J. Evans (2003), Global inventory of nitrogen oxide emissions constrained by space-based observations of $\mathrm{NO}_{2}$-columns, J. Geophys. Res., 108(D17), 4537, doi:10.1029/2003JD003453.

$\mathrm{Nüß,} \mathrm{J.} \mathrm{H.} \mathrm{(2005),} \mathrm{An} \mathrm{improved} \mathrm{tropospheric} \mathrm{NO}_{2}$ retrieval from GOME and SCIAMACHY measurements, Ph.D. thesis, Univ. of Bremen, Bremen, Germany.

Olivier, J. G. J., J. J. M. Berdowski, J. A. H. W. Peters, J. Bakker, A. J. H. Visschedijk, and J.-P. J. Bloos (2001), Applications of EDGAR, including a description of EDGAR 3.0: Reference database with trend data for 1970-1995, RIVM Rep. 773301 001, RIVM, Bilthoven. Netherlands.

Platt, U., D. Perner, and H. W. Pätz (1979), Simultanous measurements of atmospheric $\mathrm{CH}_{2} \mathrm{O}, \mathrm{O}_{3}$ and $\mathrm{NO}_{2}$ by differential optical absorbtion, J. Geophys. Res., 84, 6329-6335.

Press, W. H., S. A. Teukolsky, W. T. Vetterling, and B. P. Flannery (1997), Numerical Recipes in C, The Art of Scientific Computing, Cambridge Univ. Press, New York.

Richter, A., and J. P. Burrows (2002), Tropospheric $\mathrm{NO}_{2}$ from GOME measurements, Adv. Space Res., 29, 1673-1683. 
Richter, A., J. P. Burrows, H. Nuess, C. Granier, and U. Niemeier (2005), Increase in tropospheric nitrogen dioxide over China observed from space, Nature, 437, 129-132, doi:10.1038/nature04092.

Savage, N. H., K. S. Law, J. A. Pyle, A. Richter, H. Nüß, and J. P. Burrows (2004), Using GOME $\mathrm{NO}_{2}$ satellite data to examine regional differences in TOMCAT model performance, Atmos. Chem. Phys., 4, 1895-1912.

Storch, H. V., and F. W. Zwiers (1999), Statistical Analysis in Climate Research, Cambridge Univ. Press, New York.

Velders, G. J. M., C. Granier, R. W. Portmann, K. Pfeilsticker, M. Wenig, T. Wagner, U. Platt, A. Richter, and J. P. Burrows (2001), Global tropospheric $\mathrm{NO}_{2}$ column distributions: Comparing 3-D model calculations with GOME measurements, J. Geophys. Res., 106(12), 12,643-12,660.

Wenig, M. (2001), Satellite measurements of long-term global tropospheric trace gas distributions and source strengths, Ph.D. thesis, Univ. of Heidelberg, Heidelberg, Germany. (Available at http://mark-wenig.de/ diss_mwenig.pdf)
World Meteorological Organization (WMO) 1957, Meteorology-A threedimensional science: Second session of the commission for aerology, WMO Bull., IV(4), 134-138.

S. Beirle, U. Platt, and T. Wagner, IUP, University of Heidelberg, D-69120 Heidelberg, Germany.

J. P. Burrows and A. Richter, IUP, University of Bremen, D-28344 Bremen, Germany.

C. D. Elvidge, National Geophysical Data Center, NOAA, Boulder, CO 80305, USA.

F. Rohrer, O. Stein, N. Toenges-Schuller, and A. Wahner, ICG-II, Forschungszentrum Jülich, D-52425 Jülich, Germany. (f.rohrer@fzjuelich.de; n.toenges@fz-juelich.de) 